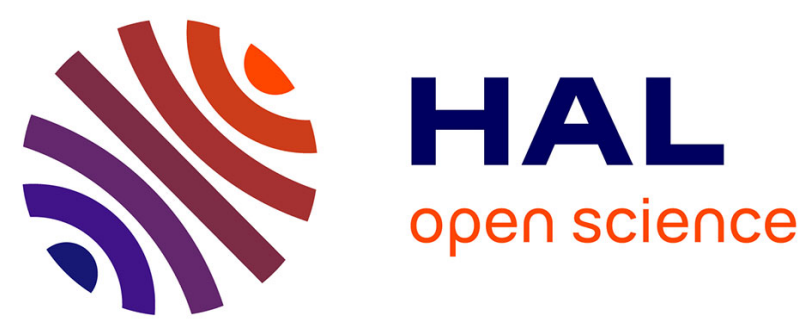

\title{
L'ethnogenèse des Frisons
}

Jens Schneider

\section{- To cite this version:}

Jens Schneider. L'ethnogenèse des Frisons. Revue du Nord, 2011, 93 (391-392), pp.749-759. hal00701557

\section{HAL Id: hal-00701557 \\ https://hal.science/hal-00701557}

Submitted on 28 Jun 2021

HAL is a multi-disciplinary open access archive for the deposit and dissemination of scientific research documents, whether they are published or not. The documents may come from teaching and research institutions in France or abroad, or from public or private research centers.
L'archive ouverte pluridisciplinaire HAL, est destinée au dépôt et à la diffusion de documents scientifiques de niveau recherche, publiés ou non, émanant des établissements d'enseignement et de recherche français ou étrangers, des laboratoires publics ou privés. 


\section{JENS SCHNEIDER*}

\section{L'ethnogenèse des Frisons}

Que dire de plus des Frisons devant une bibliographie aussi vaste que variée, comprenant notamment l'œuvre de Stéphane Lebecq d'un côté et le Handbuch des Friesischen de l'autre ${ }^{1}$ ? Depuis les années 1980, de nombreuses études archéologiques, historiques et linguistiques ont revu le passé frison sous tous les angles. Par rapport à une recherche française et belge qui s'est peut-être davantage intéressée aux activités économiques, notamment autour de la question d'un commerce à longue distance ${ }^{2}$, les travaux en Allemagne et surtout aux Pays-Bas ont cherché à montrer les implications idéologiques considérables dont les études sur les Frisons ont fait l'objet dans le passé. Après les Germains et les Gaulois ou les Celtes, les Frisons semblent être la dernière gens sur laquelle on projette la représentation d'hommes libres ayant développé et maintenu leur mode de vie, leur système juridique et leur langue à l'écart des grandes transformations des $\mathrm{IV}^{\mathrm{e}}-\mathrm{VIII}{ }^{\mathrm{e}}$ siècles. Aujourd'hui encore, l'expression de « Frison libre » (vrije fries) ${ }^{3}$ et la singularité prêtée au droit frison restent des arguments forts dans les discussions sur les origines des Frisons.

En deux mots, l'idée toujours défendue est celle d'un peuple frison qui, depuis la plus haute Antiquité, aurait vécu durant des siècles dans une sorte d'état de grâce, sans avoir subi d'influences durables par le contact d'autres gentes. Les Frisons auraient donc connu un processus d'ethnogenèse atypique, sans avoir eu à se distinguer par rapport à d'autres groupes. Cette évolution sans acculturation s'expliquerait par une sorte de splendid isolation que les Frisons auraient connue sur leurs terpen, ces îlots artificiels des rivages de la mer du Nord ${ }^{4}$.

*. - Jens SCHNEIDER, ingénieur de recherche, Université Paris-Est Marne-la-Vallée, EA 3350 ACP, 5 Boulevard Descartes, 77454 Marne-la-Vallée cedex 2.

1. - Handbuch des Friesischen. Handbook of Frisian Studies, H. H. Munske éd., Tübingen, 2001.

2. - G. DESPY, «Villes et campagnes aux IX et $\mathrm{X}^{\mathrm{e}}$ siècles: l'exemple du pays mosan », Revue du Nord, t. 50, 1968, p. 145-168; D. HÄGERMANN, « Grundherrschaft und städtischer Besitz in urbarialen Quellen des 9. Jahrhunderts (Saint-Maur-des-Fossés, Saint-Remi de Reims und Saint-Amandles-Eaux) » dans Villes et campagnes au Moyen Âge, Mélanges Georges Despy, J.-M. DuvosQuEL et A. Dierkens éd., Liège, 1991, p. 355-365.

3. - Intitulé d'un périodique annuel édité par l'Académie frisonne (Fryske Akademy) au Pays-Bas: http://www.fryske-akademy.nl/fa/utjeften/de-vrije-fries (consulté le 15/12/10).

4. - Voir la carte dressée par P. C. J. A. BoELES, Friesland tot de elfde eeuw. Zijn voor-en vroege geschiedenis, La Haye, 1951. 
Le Friesland désigne aujourd'hui une province administrative néerlandaise et deux régions allemandes qui longent la côte à l'Ouest et au nord de Hambourg. À l'heure actuelle, être frison représente une revendication identitaire, mais dont la dimension historique doit être interrogée. C'est ce qui nous conduit à esquisser ici quelques réflexions sur l'ethnogenèse et la glottogenèse frisonnes ${ }^{5}$.

\section{Les gentes du haut Moyen Âge}

Le premier, Reinhard Wenskus a parlé de Traditionskern, devenu célèbre depuis lors, pour désigner le noyau identitaire autour duquel se sont construites les gentes, tribus ou peuples, du haut Moyen Âge ${ }^{6}$. Pour cet historien, ce noyau de tradition peut être considéré comme rassemblé lors de l'institution d'un roi militaire; la gens qui se donne un roi devient alors un groupe ethnique. C'est le processus qu'auraient connu les Germains, les Goths, les Lombards, les Vandales ou bien les Frisons. Avec le concept d'ethnogenèse, développé ensuite par Herwig Wolfram et ses élèves, la perspective biologiste partagée par les historiens allemands jusqu'aux années 1960 devint franchement obsolète ${ }^{7}$. En se fondant sur les travaux de Reinhard Wenskus, les chercheurs viennois ont montré que les groupes dits ethniques étaient en fait des ensembles qui se définissaient eux-mêmes ${ }^{8}$. Ce n'est donc pas l'ascendance commune qui fait la gens, mais l'identité qu'elle se donne et qui, elle, peut contenir un élément mythique décrivant des origines communes ${ }^{9}$. L'idée selon laquelle une mémoire collective constitue et stabilise un groupe avait déjà été proposée par Maurice Halbwachs ${ }^{10}$. Mais la nouveauté de la théorie de l'ethnogenèse résidait dans l'analyse des processus identitaires dans la formation des groupes ethniques entre Antiquité tardive et haut Moyen Âge ${ }^{11}$.

5. - Cet essai reprend des réflexions proposées dans le cadre de ma thèse, Auf der Suche nach dem verlorenen Reich. Lotharingien im 9. und 10. Jahrhundert, Cologne, 2010, p. 194-199. Je tiens à remercier Magali Coumert pour sa relecture critique.

6. - R. Wenskus, Stammesbildung und Verfassung. Das Werden der frühmittelalterlichen gentes, Cologne, 1961 ( $2^{\mathrm{e}}$ éd. 1977).

7. - Strategies of Distinction. The Construction of Ethnic Communities 300-800, W. PoHL et H. Reimitz éd., Leyde, 1998; W. Pohl, « Aux origines d'une Europe ethnique. Transformations d'identités entre Antiquité et Moyen Âge », Annales, t. 60, 2005, p. 183-208. Résumé épistémologique par W. PoHL, « Modern Uses of Early Medieval Ethnic Origins » dans Usages et Mésusages du Moyen Age du XIX $X^{e}$ au XX $X^{e}$ siècle, J. M. BAK et al. éd., Munich, 2009, p. 55-70.

8. - G. M. BERNDT, Konflikt und Anpassung. Studien zu Migration und Ethnogenese der Vandalen, Husum, 2007, p. 23.

9. - M. COUMERT, Origines des peuples : les récits du Haut Moyen Âge occidental (550-850), Paris, 2007 ; A. Plassmann, Origo gentis. Identitäts- und Legitimitätsstiftung in früh- und hochmittelalterlichen Herkunftserzählungen, Berlin, 2007.

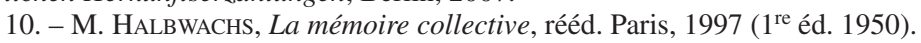

11. - R. Wenskus, Stammesbildung, op. cit. (n. 6); H. Wolfram, « Origo et religio. Ethnische Traditionen und Literatur in frühmittelalterlichen Quellen » dans Mittelalter. Annäherungen an eine fremde Zeit, W. HARTMANN éd., Ratisbonne, 1993, p. 27-39; Die Suche nach den Ursprüngen. Von der Bedeutung des frühen Mittelalters, W. PoHL éd., Vienne, 2004. 
Autrement dit, pendant cette longue phase de transition, un peuple, ou une gens, a d'abord été déterminée par une représentation identitaire construite au sein du groupe dont elle devint ensuite la base d'une conscience commune.

La théorie de l'ethnogenèse a marqué la recherche sur le très haut Moyen Âge bien au-delà du cercle viennois rassemblé autour d'Herwig Wolfram puis de Walter Pohl, même si l'adaptation de ce modèle au niveau international ne s'est pas faite sans critique. En Allemagne, le concept a été repris assez tôt par Jörg Jarnut ou Joachim Ehlers ${ }^{12}$; il a été suivi plus tard, et peut-être avec davantage de réserves, en France, comme en témoignent la thèse de Magali Coumert ${ }^{13}$ et la tenue du colloque « Ethnicité et Identité », à Caen en 2004, au cours duquel fut posée par le regretté Yves Modéran la question de la pertinence du modèle de l'ethnogenèse appliqué à l'Afrique du Nord, « des Maures aux Berbères ${ }^{14}$. Le terme d'ethnogenèse invite en effet à la réflexion: pris à la lettre, il ne signifie rien d'autre que la formation d'un groupe ethnique alors même que ses promoteurs postulaient la négation de l'ethnicité des groupes en question, considérés selon Reinhard Wenskus comme des communautés de tradition (Traditionsgemeinschaften). Une autre critique porte sur le problème de l'homogénéité des peuples en migration ${ }^{15}$. À cela s'ajoute la mise en cause fondamentale du critère ethnique attribué selon les données de la culture matérielle recueillies par les archéologues ${ }^{16}$.

Le terme d'ethnogenèse est aujourd'hui de moins en moins utilisé. Reste l'approche méthodologique désormais admise par tous les historiens qui ne voient plus les peuples du haut Moyen Âge définis en fonction de leurs origines biologiques ou de leur appartenance linguistique, mais par un ensemble de traditions collectives dont la construction participe à la formation de l'identité ethnique ${ }^{17}$.

12. - J. JARNUT, «Aspekte frühmittelalterlicher Ethnogenese in historischer Sicht » dans ID., Herrschaft und Ethnogenese im Frühmittelalter, Münster, 2002, p. 19-27 (rééd. d'un article publié en 1985); J. EHLERS, "Schriftkultur, Ethnogenese und Nationsbildung », Frühmittelalterliche Studien, t. 23, 1989, p. 302-317. Cf. A. A. Lund, Die ersten Germanen. Ethnizität und Ethnogenese, Heidelberg, 1998.

13. - M. COUMERT, Origines, op. cit. (n. 9).

14. - Ethnicité et Identité. Concepts, débats historiographiques, exemples (III ${ }^{e}$ XII ${ }^{e}$ siècle), V. GAZEAU et al. éd., Caen, 2008.

15. - On Barbarian Identity. Critical Approaches to Ethnicity in the Early Middle Ages, A. GILlETT éd., Turnhout, 2002; en dernier lieu M. COUMERT et B. DuMÉZIL, « Les 'grandes migrations' et la construction des identités $\left(\mathrm{IV}^{\mathrm{e}}-\mathrm{VI}^{\mathrm{e}}\right.$ siècle): problème d'histoire ou d'historiographie? »dans Des sociétés en mouvement. Migrations et mobilité au Moyen Âge, actes du $40^{\mathrm{e}}$ congrès de la SHMESP (Nice, 2009), Paris, 2010, p. 33-44.

16. - S. BRATHER, Ethnische Interpretationen in der frühgeschichtlichen Archäologie. Geschichte, Grundlagen und Alternativen, Berlin, 2004.

17. - W. PoHL, « Telling the Difference: Signs of Ethnic Identity » dans Strategies of Distinction, op. cit. (n. 7), p. 17-69; M. COUMERT et B. DuMÉzIL, «Les 'grandes migrations' », op. cit. (n. 15), p. 35 . 


\section{Une ethnicité difficile à établir}

L'ethnicité des Frisons est difficile à cerner, même lorsque notre documentation les concernant s'étoffe, à partir du tournant des VIII ${ }^{\mathrm{e}}$-IX ${ }^{\mathrm{e}}$ siècle. La question de l'origine des fameux pallia fresonica envoyés par Charlemagne en Perse montre la complexité du sujet ${ }^{18}$. L'expression suppose que les contemporains ont associé ces étoffes aux marchands frisons qui en ont donc au moins assuré la commercialisation ${ }^{19}$. De son côté, Ermold le Noir évoque le vin vendu aux Frisons ${ }^{20}$; et Loup de Ferrières rapporte l'histoire d'un marchand frison nommé Ibbo au service de l'abbaye de Saint-Maximin de Trèves ${ }^{21}$. Bien que le diplôme de Louis le Pieux qui confirme le tonlieu sur les negotiatores vel artifices seu et Frisiones donné le 11 septembre 829 pour l'Église de Worms paraisse au moins interpolée ${ }^{22}$, il reste convenu que le terme Frison était alors utilisé comme synonyme de celui de marchand ${ }^{23}$. D'un autre côté, il ne faut pas oublier que les Frisons étaient également connus comme formant une gens distincte, voisine des Saxons et des Danois $^{24}$, active en Angleterre ${ }^{25}$, et dont le territoire, pendant un moment, fut revendiquée à la fois par les rois francs et danois ${ }^{26}$.

La Lex Frisionum nous renseigne aussi sur les contrées considérées comme frisonnes ${ }^{27}$. Il s'agit d'une Frise en trois parties qui se déduit des différentes

18. - Notker Balbulus, Taten Kaiser Karls des Großen, H.-F. HAEFELE éd., Berlin, 1959 (MGH, $S R G$, n.s., 12), II, 9, p. 63.

19. - H. PirenNe, « Draps de Frise ou draps de Flandre? Un petit problème d'histoire économique à l'époque carolingienne », Vierteljahresschrift für Sozial- und Wirtschaftsgeschichte, t. 7, 1909, p. 308-315; S. LEBECQ, Marchands et navigateurs frisons du haut Moyen Age, Lille, 1983, vol. 1, p. 131-134; ID., « Friesenhandel » dans Reallexikon der Germanischen Altertumskunde, t. 10, 1996, p. $69-80 ;$ P. SCHMID, ibid., p. 58.

20. - Ermold LE NoIr, Poème sur Louis le Pieux et Épîtres au roi Pépin, E. FARAL éd., Paris, 1932, v. $119-120$, p. 210.

21. - Loup DE FERRIÈRES, Vita Maximini episcopi Trevirensis, B. KRUSCH éd., dans MGH, Scriptores rerum Merovingicarum, t. 3, Hanovre, 1896, ch. 19, p. 80-81.

22. - Die Regesten des Kaiserreichs unter den Karolingern 751-918, J. F. BÖHMER et E. MüHLbaCher éd., Hildesheim, 1966 (Regesta Imperii, 1), n 871. En attendant l'édition par les soins de T. Kölzer, le texte du diplôme doit être reconstruit d'après une confirmation par Otton $\mathrm{I}^{\mathrm{er}}$ : Die Urkunden Konrad I., Heinrich I. und Otto I., T. SiCKEL éd. (MGH, Diplomata), Munich, 1980, $\mathrm{n}^{\circ}$ 84, p. 165; cf. T. KöLZER, Kaiser Ludwig der Fromme (814-840) im Spiegel seiner Urkunden, Paderborn, 2005, $\mathrm{n}^{\circ} 281$ du tableau.

23. - S. LEBECQ, Marchands et navigateurs, op. cit. (n. 19), vol. 1, p. 16; ID., « Frisons et Vikings. Remarques sur les relations entre Frisons et Scandinaves aux VII ${ }^{\mathrm{e}}-\mathrm{IX}^{\mathrm{e}}$ siècles » dans Les mondes normands (VII ${ }^{e}$-XII ${ }^{e}$ siècle), H. GALINIÉ éd., Caen, 1989, p. 45-59.

24. - Voir l'analyse des mentions des Annales regni Francorum, Bertiani, Fuldenses et Xantenses dans J. SCHNEIDER, Auf der Suche, op. cit. (n. 5), p. 78-91 et annexe 3, p. 498-499; K. VAN VLIET, In kringen van kanunniken. Munsters en kapittels in het bisdom Utrecht 695-1227, Zutphen, 2002, p. 133-138.

25. - S. LEBECQ, Marchands et navigateurs, op. cit. (n. 19), vol. 1, p. 89-92.

26. - Éginhard, Vie de Charlemagne, L. Halphen éd., Paris, 1967, ch. 14, p. 40-42: rex Godofridus (...) Frisiam quoque atque Saxoniam haud aliter atque suas provincias aestimabat.

27. - Lex Frisionum, K. A. et A. EcKhardt éd. (MGH, Fontes iuris Germanici antiqui, t. 12), Hanovre, 1982; H. SIEMS, « Lex Frisionum » dans Handwörterbuch zur Rechtsgeschichte, t. 2, 
nuances que connaissait le droit, avec, au centre, « l'Oostergo (...) qui constituait avec le Westergo le vieux cœur de la Frise » au nord-est de l'actuel IJsselmeer $^{28}$. Malheureusement, on ne saurait attribuer la composition de la Lex Frisionum à un moment précis du $\mathrm{VIII}^{\mathrm{e}}$ ou du $\mathrm{IX}^{\mathrm{e}}$ siècle car la date et les conditions de sa rédaction sont loin d'être certaines. En tout cas, elle ne précise pas ce qui fondait l'appartenance à la gens Frisionum puisque, logiquement, elle en suppose l'existence. Comparée à d'autres leges barbarorum, elle révèle d'ailleurs des éléments communs, notamment avec la Lex Alamannorum et la Lex Thuringorum.

Dans une perspective traditionnelle, on insistait sur les rapports entretenus par le Frison avec la mer. Il avait beau être marchand, l'idée selon laquelle il avait domestiqué la mer du Nord constituait le cœur d'une véritable idéologie frisonne ${ }^{29}$. Les recherches archéologiques (et leurs interprétations) ont soutenu cette hypothèse d'une continuité ethnique reposant sur la familiarité avec l'élément marin. La construction de digues fut aussi considérée comme un savoir-faire spécifiquement frison. Le mot français « digue » tout comme l'allemand «Deich» ou le néerlandais « dijk » proviennent du mot vieux-frison ou anglo-saxon $d \overline{i c}^{30}$. Les terpen représentent un autre élément considéré comme typiquement frison par les archéologues : on désigne ainsi des établissements de caractère agricole érigés sur des buttes dans la zone argileuse de l'estran qui offrait de riches pâtures au bétail ${ }^{31}$.

L'idée selon laquelle le peuple frison aurait développé, au plus tard depuis l'époque romaine, des techniques spécifiques d'occupation du sol dans cette zone située entre terre et mer perd de sa force depuis une quinzaine d'an-

27. - (suite) 1978, col. 1916-1922; S. LEBECQ, « La Lex Frisionum », à paraître dans Les lois barbares, B. DuMÉZIL et al éd.

28. - S. LEBECQ, « Les Vikings en Frise: chronique d'un échec relatif » dans Les fondations scandinaves en Occident et les débuts du duché de Normandie, P. BAuduin éd., Caen, 2005, p. 97-112.

29. - J. GRIMM, Geschichte der deutschen Sprache, Leipzig, 1868 ( $1^{\text {re }}$ éd. 1848), vol. 2, ch. XXIV, p. 464-473; M. ERBE, Belgien, Niederlande, Luxemburg. Geschichte des niederländischen Raumes, Stuttgart, 1993, p. 12-13; D. P. BLoK et H. HINZ, « Friesen, Friesland (A.) » dans Lexikon des Mittelalters, t. 4, 1989, p. 970-971; E. TAAYKE et E. KNOL (II § 14), D. STRAUCH (III § 24), «Friesen » dans Reallexikon der Germanischen Altertumskunde, t. 10, 1996, p. 35-37 et 64. Premier état des lieux critique par P. BoEles, Friesland tot de elfde eeuw, op. cit. (n. 4), p. 559-592 en attendant W. VAN EGMOND, « Utrecht in the Early Middle Ages : historical research and problems », sous presse.

30. - F. Kluge, Etymologisches Wörterbuch der deutschen Sprache, 24e édition par E. SEEBOLD, Berlin, 2002, p. 185; Le nouveau Petit Robert, J. ReY-Debove et A. ReY éd., Paris, 1996, p. 645.

31. - E. Kramer et E. TAAYKe, « Friesen entlang der Nordseeküste von 400 bis $1000 \mathrm{n}$. Chr. » dans Friesen, Sachsen und Dänen. Kulturen an der Nordsee, 400 bis 1000 n. Chr., J. DöRING et al. éd., Leeuwarden, 1996, p. 9-23. Pour la situation géologique voir De ondergrond van Nederland, E. F. J. DE MULDER et al. éd., Groningen, 2003; http://www.geo.uu.nl/fg/palaeogeography (consulté le $15 / 12 / 10)$. 
nées $^{32}$. La relecture des fouilles archéologiques montre que la construction de digues a en réalité commencé à la fin du $\mathrm{x}^{\mathrm{e}}$ siècle, ce qui en fait plutôt une entreprise liée à l'Empire ottonien ${ }^{33}$. Quant aux terpen, les analyses des fouilles indiqueraient, contrairement aux interprétations plus anciennes, une rupture de l'occupation humaine pendant une douzaine de générations à partir du $\mathrm{IV}^{\mathrm{e}}$ siècle $^{34}$.

Cette réévaluation de la culture matérielle dans l'espace frison a été provoquée par un ouvrage publié en 1951 qui a mis en cause une bonne partie des connaissances sur les origines frisonnes. Pieter Boeles, juriste de formation, travaillait sur ces problèmes depuis les années 1920. Il faut noter que la première édition de son livre n'a pas suscité beaucoup d'intérêt tandis que la deuxième, revue et augmentée, publiée dix ans avant le livre de Reinhard Wenskus, a fait scandale, tellement le sujet était lié (et l'est toujours) à l'identité frisonne et néerlandaise ${ }^{35}$. À en croire Jos Bazelmans, Pieter Boeles aurait été en quelque sorte excommunié pour cette raison ${ }^{36}$. En reprenant la réflexion de Pieter Boeles, Jos Bazelmans a présenté dans deux articles, non sans polémique, les arguments archéologiques et historiographiques de la question $^{37}$. En ce qui concerne la culture matérielle, il n'y a pas eu de fouilles révolutionnaires qui auraient fondamentalement changé les interprétations. Le constat que la construction des maisons, la céramique et les fibules après le $\mathrm{V}^{\mathrm{e}}$ siècle se distinguent nettement de celles de l'époque romaine repose surtout sur l'interprétation des fouilles. Or Jos Bazelmans note que, pendant la plus grande partie du $\mathrm{XX}^{\mathrm{e}}$ siècle, l'analyse archéologique aux Pays-Bas était marquée, comme en Allemagne d'ailleurs, par une compréhension particulière de l'ethnicité et de l'âme d'un peuple (Volksgeist). Toujours selon Jos Bazelmans, la tradition écrite témoigne d'une lacune d'environ trois siècles qui aurait été ignorée par les défenseurs de la continuité frisonne. Entre un panégyrique dédié à Constance Chlore à la fin du $\mathrm{III}^{\mathrm{e}}$ siècle et les attestations $\mathrm{du} \mathrm{VII}^{\mathrm{e}}$ siècle, les sources ignorent les Frisons: on ne trouve aucune mention d'officiers d'origine frisonne dans l'armée romaine dans le contexte des affrontements militaires du $v^{\mathrm{e}}$ siècle, ni aucune allusion chez Grégoire de

32. - J. Bazelmans, « Sijn de Friezen wel Friezen? », Spiegel historiael, t. 33, 1998, p. 197-201; ID., D. Gerrets, J. DE Koning et P. Vos, «Zoden aan de dijk: kleinschalige dijkbouw in de late prehistorie en protohistorie van noordelijk Westergo », De Vrije Fries, t. 78, 1999, p. 7-74; J. BAZELMANS, «Is Frieslands oudheid tegenwoordig zonder belang? », ibid., t. 82, 2002, p. 302-309.

33. - E. Kramer et E. TAAYKe, «Friesen entlang der Nordseeküste », op. cit. (n. 31), p. 23 ; Handbuch des Friesischen, op. cit. (n. 1), p. 487-492 (J. M. Bos) et, déjà, S. LEBECQ, Marchands et navigateurs, op. cit. (n. 19), p. 119-125.

34. - J. Bazelmans, «Sijn de Friezen wel Friezen? », op. cit. (n. 32); J. M. Bos dans Handbuch des Friesischen, op. cit. (n. 1), p. 490: «Certainly there no longer was a functioning Frisian society ». 35. - P. C. J. A. Boeles, Friesland tot de elfde eeuw. Zijn oudste beschaving en geschiedenis, La Haye, $1927 ; 2^{\mathrm{e}}$ édition 1951 (titre modifié, voir n. 4).

36. - J. BAZELMANS, « Is Frieslands oudheid tegenwoordig zonder belang ? », op. cit. (n. 32), p. 302. 37. - Voir supra n. 32. 
Tours $^{38} . \mathrm{Au} \mathrm{VI}^{\mathrm{e}}$ siècle, les témoignages de Procope et de Venance Fortunat seraient trop sommaires et imprécis pour être pris en compte. Venance Fortunat évoque dans un chant ad Chilpericum regem les Frisons parmi les peuples qui redoutent Chilpéric $\mathrm{I}^{\mathrm{er}}$ : Terror [es] extremis Fresonibus atque Suebis ${ }^{39}$. Procope désigne dans son Histoire des guerres contre les Vandales, les Goths et les Perses trois peuples qui habitent la (Grande-)Bretagne, chacun ayant un roi propre: les Angles, les Phrisones ( $\phi \rho \sigma_{\sigma \sigma \sigma \nu} \in \zeta$ ) et les Bretons $^{40}$. Jos Bazelmans considère que, écrivant à Ravenne et à Constantinople, ces auteurs étaient trop loin pour pouvoir juger de l'existence d'une gens frisonne. Le fait qu'ils énumèrent les Frisons avec les Suèves ou bien parmi les Angles et les Bretons montrerait qu'ils ne disposaient que d'informations de deuxième ou de troisième main. Les contemporains du $\mathrm{VI}^{\mathrm{e}}$ siècle s'attendaient à ce que les Frisons soient nommés quand il était question de peuples barbares ${ }^{41}$. Depuis Pline ou Tacite en effet, les Frisons passaient pour être de redoutables combattants, ce qui explique pourquoi leurs vainqueurs romains ne manquaient pas de les mentionner ${ }^{42}$.

En somme, les Frisons tels qu'ils apparaissent dans les sources du haut Moyen Âge se présentent comme une gens relativement jeune: un groupe qui se serait installé sur la côte plus ou moins dépeuplée de la mer du Nord et qui reprit le nomen gentilis frison, peu importe que cette appropriation ait été volontaire ou qu'elle lui ait été attribuée parce qu'il était établi dans cette région côtière. Cette gens se fit surtout connaître dans les sources avec le roi Radbod $^{43}$. Après de nombreux conflits militaires avec les Francs à la fin du $\mathrm{VII}^{\mathrm{e}}$ et au début du $\mathrm{VIII}^{\mathrm{e}}$ siècle, les Frisons durent céder une partie de leur territoire, ce que les Francs célèbrent comme un succès de Pépin II contre ce même dux Radbod ${ }^{44}$. Dans la perspective de l'ethnogenèse, les Frisons formeraient donc un peuple constitué au cours du $\mathrm{VII}^{\mathrm{e}}$ siècle et qui aurait ravivé la désignation prestigieuse de Frisons en s'en servant comme élément identitaire. L'usage de ce terme ethnique par les Francs aurait contribué à cette évolution.

38. - Toutes les références sont rassemblées par E. SEEBOLD dans Handbuch des Friesischen, op. cit. (n. 1), p. 479-487; cf. P. BoEles, Friesland tot de elfde eeuw, op. cit. (n. 4), p. 269-287.

39. - Chant 9 dans l'édition de F. Leo, Venanti Honori Clementiani Fortunati presbyteri Italici Opera poetica, dans $M G H$, AA, t. 4-1, Berlin, 1881; extrait reproduit et présenté par S. LEBECQ, Marchands et navigateurs, op. cit. (n. 19), vol. 2, p. 17-18.

40. - Ibid., p. 225-227.

41. - J. Bazelmans, «Sijn de Friezen wel Friezen?», op. cit. (n. 32), p. 200: «En de Friezen behoorden daartoe, zeker voor geleerde mensen zoals Fortunatus die het werk van Tacitus uit de eerste hand kende. »

42. - S. LEBECQ, «Friesenhandel », op. cit. (n. 19); B. SJÖLIN, « Friesen », § 2-9, dans Reallexikon der Germanischen Altertumskunde, t. 10, 1996, p. 6-14.

43. - W. S. VAN EGMOND, « Radbod van de Friezen, een aristocraat in de periferie », Millenium, t. 19, 2005 , p. 24-44.

44. - Liber historiae Francorum, B. KRUSCH éd., dans MGH, Scriptores rerum Merovingicarum, t. 2, Hanovre, 1888 , ch. 49-50, p. 323-325. 
Ce point de vue, défendu actuellement par Jos Bazelmans et d'autres, enlève à la société frisonne médiévale son ancienneté particulière. Si les Frisons ne sont pas plus anciens et «pur-sang » ${ }^{45}$ que les autres, un élément identitaire revendiqué par une partie considérable de la population des PaysBas et de l'Allemagne du Nord-ouest disparaît. À cela s'ajoute que le droit frison, à la différence de la Lex Frisionum, est une collection de sources vernaculaires fixée sans influence franque et ne date que du XIII ${ }^{\mathrm{e}}$ siècle $^{46}$. Par conséquence, la fameuse tripartition en trois sphères juridiques qui y apparaît n'est plus forcément un héritage datant des premiers siècles de notre ère. Elle pourrait s'expliquer par la structure sociale hétérogène de la population des régions côtières établie entre l'estuaire de l'Escaut, à l'ouest, et celui de l'Ems, à l'est.

Ainsi, à côté de la faiblesse de l'argumentaire archéologique et ethnologique, les interprétations tirées des sources juridiques apparaissent bien fragiles. En dernier recours, l'approche linguistique peut-elle apporter des éclaircissements?

\section{La langue frisonne}

L'hétérogénéité qui se manifeste dans la tradition du droit frison se retrouve également dans la situation linguistique. La division remarquable du vieux-frison en différents parlers n'est justement pas considérée comme le résultat d'une différenciation en plusieurs variantes linguistiques mais comme existant dès les origines ${ }^{47}$. La tripartition linguistique serait inhérente à la glottogenèse frisonne. Traditionnellement, on suppose l'évolution d'un frison primitif à partir du germanique occidental (ou «westique ») au $\mathrm{VIII}^{\mathrm{e}}$ siècle au plus tard ${ }^{48}$. Le mot germanique est compris ici exclusivement comme un terme regroupant plusieurs langues apparentées et non pas comme déterminant une qualité ethnique ${ }^{49}$. Toute observation sur la genèse de la langue frisonne reste en quelque sorte hypothétique étant donnée l'extrême pauvreté des témoignages écrits. La Lex Frisionum, texte plus ou moins carolingien, contient une vingtaine d'expressions vernaculaires difficiles à dater ${ }^{50}$. Les traditions runiques sont conservées dans une vingtaine d'inscrip-

45. - J. BAZELMANS, «Sijn de Friezen wel Friezen?», op. cit. (n. 32), p. 198.

46. - Altfriesische Rechtsquellen. Texte und Übersetzungen, W. J. BuMA et W. EBEL éd., Göttingen, 1963-1977; B. SCHNEIDMÜLLER, « Friesen - Welfen - Braunschweiger. Träger regionaler Identität im 13. Jahrhundert » dans Identité régionale et conscience nationale en France et en Allemagne du Moyen Âge à l'époque moderne, R. BABEL et J.-M. MoEgLin éd., Sigmaringen, 1997, p. 305-324. 47. - B. SJÖLIN, « Friesen », op. cit. (n. 42), p. 7 : « vom ersten Anfang an ».

48. - Ibid.; H. KRAHE et W. MEID, Germanische Sprachwissenschaft, t. 1, Einleitung und Lautlehre, Berlin, 1969 et les contributions de H. F. NIELSEN et N. ÅRHAMMAR dans Handbuch des Friesischen, op. cit. (n. 1), p. 512-523 et 531-537.

49. - J. JARNUT, « Germanisch. Plädoyer für die Abschaffung eines obsoleten Zentralbegriffes der Frühmittelalterforschung » dans Die Suche nach den Ursprüngen, op. cit. (n. 11), p. 107-113. 50. - Voir supra n. 29-30 ; P. BoELES, Friesland tot de elfde eeuw, op. cit. (n. 4), p. 469-478. 
tions datées entre le $\mathrm{VI}^{\mathrm{e}}$ et le début du $\mathrm{IX}^{\mathrm{e}}$ siècle; la plus ancienne, qui remonte peut-être même au $\mathrm{V}^{\mathrm{e}}$ siècle, contient deux signes runiques communément transcrits par $l w^{51}$. Une partie de ces inscriptions repose sur le système runique anglo-frison Futhork, plus récent que le Futhark, plus répandu ${ }^{52}$.

Pour cette raison, les caractéristiques du frison primitif ont été déduites du frison septentrional et méridional pourtant beaucoup plus récents. On a vu dans le texte conservé du droit frison - qui date, on l'a dit, du XIII ${ }^{\mathrm{e}}$ siècle l'état linguistique le plus ancien du vieux-frison. Ne sachant pas quand ce vieux-frison s'est constitué comme une langue propre ni comment il a évolué par rapport au germanique, on le considère comme un reliquat du germanique de la mer du Nord (nordseegermanisch ou ingvaeonique). Il faut alors inférer les détails de la mutation phonétique qui a dû se dérouler entre le stade d'évolution du germanique de la mer du Nord, suffisamment connu par d'autres langues qui en sont issues, et le vieux-frison, tel qu'il est connu au XIII ${ }^{\mathrm{e}}$ siècle. On ne s'étonnera pas de lire dans le Reallexikon der Germanischen Altertumskunde que la chronologie relative de cette évolution est fort problématique et fait l'objet d'un débat scientifique ${ }^{53}$ !

Ce qui est relativement certain concerne les traits communs dans l'évolution des voyelles avec l'anglo-saxon (insulaire), non pas avec le vieux-saxon. Au niveau des consonnes, il semble qu'il n'y ait guère d'évolutions à constater par rapport au germanique occidental, ce qui est également le cas avec le vieux-saxon. D'autres langues émanant du germanique occidental ont connu davantage de mutations pour devenir, par exemple, le vieux-francique, la langue maternelle de Pépin et de Charlemagne. Si on s'interroge sur la communauté linguistique, c'est-à-dire les hommes qui s'exprimaient en vieux-frison, rien n'indique qu'elle remonte au temps du germanique occidental et qu'elle ait évolué de façon isolée, sur les terpen, au cours des siècles. Il est au contraire bien imaginable que les membres de la future communauté frisonne, linguistique et politique, ont importé le système de consonnes propre au germanique occidental qui aurait connu par la suite peu de modifications. Dans tous les cas, il faut partir du constat d'une relation assez étroite avec la situation linguistique insulaire, sans exclure que cette relation fût plus que linguistique.

La question de la glottogenèse frisonne, ce processus dont on ne connaît que le résultat fixé par écrit au XIII ${ }^{\mathrm{e}}$ siècle, reste ouverte. À côté de la position traditionnelle du reliquat d'un parler germanique de la mer du Nord, il existe une explication alternative qui a peut-être le mérite de résoudre plusieurs pro-

51. - Ibid., p. 338-359; R. I. PAGE dans Handbuch des Friesischen, op. cit. (n. 1), p. 523-530.

52. - K. DüWEL, « Friesen », § 13, dans Reallexikon der Germanischen Altertumskunde, t. 10, 1996, p. 28-29; ID., Runenkunde, Stuttgart, 2001, p. 86-87.

53. - B. SJÖLIN, « Friesen », op. cit. (n. 42), p. 7. 
blèmes liés à cette question, aussi bien sur le plan linguistique qu'historiographique. Le vieux-frison tel qu'il se manifeste au XIII ${ }^{\mathrm{e}}$ siècle pour la première fois n'a pas forcément gardé des traits linguistiques anciens en raison d'une évolution isolée; ce qui paraît admirablement ancien peut très bien être le résultat d'une influence anglo-saxonne entre le $\mathrm{VII}^{\mathrm{e}}$ et le $\mathrm{XIII}^{\mathrm{e}}$ siècle. Nous savons aujourd'hui que les frontières naturelles n'ont jamais été étanches, loin s'en faut. La Manche et la mer du Nord étaient une zone de passage et de contacts fréquents dont tirèrent parti les « marchands et navigateurs frisons ».

Dorestad et Quentovic, étudiés par Stéphane Lebecq ${ }^{54}$, furent les points de passage de cette extrême mobilité des Frisons entre le continent et l'Angleterre. Au-delà des contacts linguistiques dans cette zone, dont témoigne le vieux-frison, il convient de s'interroger sur l'importance, pour l'ethnogenèse frisonne, du processus d'installation des Angles, des Jutes et des Saxons en Angleterre, voire sur une participation frisonne à cette installation $^{55}$. Ceci mènerait à l'hypothèse d'une ethnogenèse et glottogenèse frisonnes ayant eu lieu au même moment, donc surtout à partir du VII ${ }^{\mathrm{e}}$ siècle. Le résultat en serait une gens frisonne relativement jeune qui ne s'était pas encore entièrement constituée à l'époque carolingienne pour apparaître comme un peuple institutionnellement et linguistiquement distinct des Francs ou des Saxons voisins. C'est pourquoi leurs institutions juridiques se distinguent mal de celles que décrivent les autres leges barbarorum. Leur langue est seulement en train de se constituer avec davantage d'influences anglosaxonnes que franques. De l'Ouest à l'Est, avant comme après, cette évolution a pu privilégier différents éléments sans qu'un compromis linguistique ait homogénéisé ces particularités qui fondent la tripartition de la langue frisonne. Une évolution semblable a été proposée dans le cas du vieux-saxon ${ }^{56}$.

$\mathrm{Au}$ terme de ces réflexions qui ne forment qu'une esquisse, on reviendra sur notre point de départ: la constitution d'une entité frisonne «perçue comme telle » par les contemporains ${ }^{57}$, un peuple ou une gens en d'autres

54. - S. LEBECQ, Marchands et navigateurs, op. cit. (n. 19), vol. 1, p. 141-163; ID., « Pour une histoire parallèle de Quentovic et Dorestad » dans Villes et campagnes au Moyen Âge, op. cit. (n. 2), p. 415-428; ID., «Quentovic: un état de la question », Studien zur Sachsenforschung, t. 8, 1993, p. 73-82; ID., «L'emporium proto-médiéval de Walcheren/Domburg: une mise en perspective » dans Peasants and Townsmen in Medieval Europe. Studia in honorem Adriaan Verhulst, J.-M. Duvosquel et E. Thoen éd., Gand, 1995, p. 73-90; ID., « La Neustrie et la mer » dans La Neustrie. Les pays au nord de la Loire de 650 à 850, H. ATsma éd., Sigmaringen, 1989, t. 1, p. 405440; Quentovic. Environnement, Archéologie, Histoire, S. LEBECQ et al. éd., Villeneuve-d'Ascq, 2010 .

55. - S. LEBECQ, Marchands et navigateurs, op. cit. (n. 19), vol. 1, p. 108.

56. - T. KLEIN, Studien zur Wechselbeziehung zwischen altsächsischem und althochdeutschem Schreibwesen und ihrer sprach- und kulturgeschichtlichen Bedeutung, Göppingen, 1977, p. 522.

57. - Expression empruntée à L. GENICOT, « 'La Neustrie', pays aux contours mal définis ? » dans La Neustrie, op. cit. (n. 54), p. 25-27. 
mots, et les répercussions identitaires de ce processus historique jusqu'au $\mathrm{XX}^{\mathrm{e}}$ siècle. L'édition actuelle de la grande encyclopédie allemande Brockhaus suggère encore la continuité d'un peuple frison sur deux mille ans, depuis l'an 12 avant J.-C. plus précisément ${ }^{58}$. Dans la dernière édition du Reallexikon der Germanischen Altertumskunde, l'ouvrage de référence en trente-cinq volumes sur ce genre de question, un des auteurs de l'article «Friesen » continue de situer les origines du peuple frison à l'âge de fer, donc aux $\mathrm{VI}^{\mathrm{e}}-\mathrm{V}^{\mathrm{e}}$ siècles avant notre ère ${ }^{59}$. Néanmoins, depuis la deuxième moitié des années 1990, en France, en Allemagne et surtout aux Pays-Bas, on a commencé à relativiser la valeur des sources archéologiques, historiographiques ainsi que celles de l'histoire du droit pour notre connaissance de l'antiquité frisonne. Dans un article paru dans le catalogue de l'exposition sur les Francs en 1996, l'espace Rhine-Meuse delta, communément abrégé en RMD par les paléogéographes ${ }^{60}$, est considéré comme une zone de contacts et d'échanges entre différentes gentes dites germaniques aux IV ${ }^{\mathrm{e}}-\mathrm{V}^{\mathrm{e}}$ siècles. Parmi les nouveaux groupes issus de cet espace dynamique, ceux qui se tournèrent vers les côtes britanniques sont appelés Saxons, ceux qui pénétrèrent à l'intérieur du continent, Francs, ceux enfin qui demeurèrent dans ce vaste delta pour occuper les côtes sont perçus comme Frisons ${ }^{61}$. En somme, les arguments discutés ici rejoignent un point de vue encore hypothétique il y a une vingtaine d'années, ce qui laisse le dernier mot à son auteur: "La Frise du haut Moyen Âge se satisfera-t-elle d'une définition ethnique ? Sûrement pas ${ }^{62}$.

Mots-clés : peuples barbares, Antiquité tardive, haut Moyen Âge, histoire des langues germaniques, historiographie.

58. - Brockhaus Enzyklopädie, Leipzig, 2006, t. 10, p. 6-7.

59. - E. TAAYKE et E. KNOL, « Friesen », op. cit. (n. 29), p. 35.

60. - Voir supra n. 32.

61. - S. LEBECQ, «Franken und Friesen » dans Die Franken - Wegbereiter Europas. Vor 1500

Jahren: König Chlodwig und seine Erben, A. WIECZOREK et al. éd., Mayence, 1996, p. 338-340.

62. - ID., Marchands et navigateurs, op. cit. (n. 19), vol. 1, p. 16. 
Schneider 20/01/12 12:15 Page 12

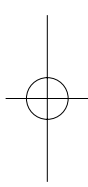

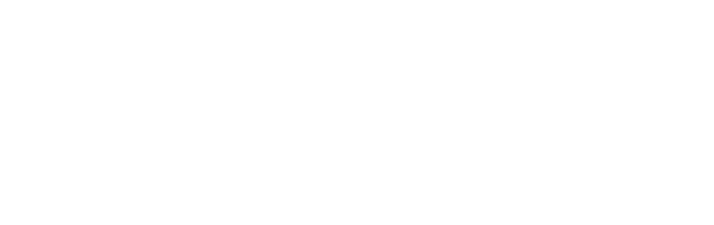

